\section{Conceptos e infraestructuras didácticas en los procesos de enseñanza-aprendizaje de la biodiversidad en Educación Infantil: análisis de la situación en Asturias} n

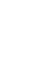

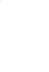

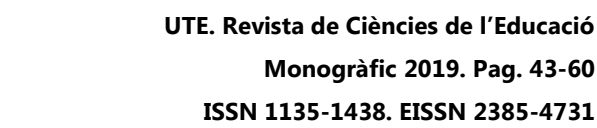

http://revistes.publicacionsurv.cat/index.php/ute

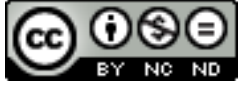

https://doi.org/10.17345/ute.2019.2

\author{
Ana Maria Segura, Antonio Torralba-Burrial \\ Rebut: 06/09/2019 Acceptat: 14/10/2019
}

\begin{abstract}
Resumen
Con el fin de conocer qué, cómo y con qué se realizan los procesos de enseñanza-aprendizaje de la biodiversidad en ambos ciclos de Educación Infantil, se ha enviado un cuestionario a los centros educativos de Asturias, siendo respondido por docentes en activo de 58 centros. El concepto de biodiversidad es generalmente manejado con un significado similar entre docentes en activo de Educación Infantil, y correspondiente con el concepto científico. En la consideración de especies amenazadas se observa en cambio una acepción mucho más restringida que la científica y legal. Los docentes manifestaron muy mayoritariamente que consideraban muy importante el estudio de la biodiversidad en esta etapa y que realizaban actividades con ese fin. Las dotaciones/infraestructuras didácticas más utilizadas en Educación Infantil para el aprendizaje de la biodiversidad han sido la pizarra digital interactiva, huerto escolar (si bien ampliamente infrautilizada) y patio con vegetación, siendo muy poco empleadas las salidas didácticas y el laboratorio escolar.
\end{abstract}

Palabras Clave: Didáctica del Medio Natural, Biodiversidad, Educación Ambiental, Educación Infantil.

\begin{abstract}
In order to know what, how and with what the teaching-learning processes of biodiversity are carried out in both cycles of Early Childhood Education, a questionnaire has been sent to the educational centers of Asturias, being answered by teachers from 58 centers. The concept of biodiversity is generally managed with a similar meaning among teachers, and corresponding to the scientific concept. In the consideration of endangered species, a much more restricted meaning than the scientific and legal one is observed instead. Teachers considered the study of biodiversity at this stage to be very important, and carried out activities for that purpose. The didactic facilities / infrastructures most used in Early Childhood Education for biodiversity learning have been the interactive whiteboard, school garden (although widely underutilized) and schoolyard with vegetation; didactic outdoor visits and the school laboratory being rarely used, instead.
\end{abstract}

Keywords: Environmental didactic, Biodiversity, Environmental Education, Early Childhood Education. 


\section{Introducción y justificación}

La Didáctica de las Ciencias Experimentales, tanto referida a la formación del profesorado como a su aplicación en las etapas educativas en las que desarrolla su futuro profesional el profesorado egresado (Educación Infantil, Educación Primaria, Educación Secundaria), está en continua transformación. Esto resulta palpable en la identidad e intensidad con la que se refieren los conceptos científicos tratados como en las metodologías, infraestructuras y recursos didácticos empleados en estos procesos de aprendizaje. Diferencias y estilos que también se muestran en diversos manuales para la formación de maestros en las últimas décadas (Garrido Romero, Perales Palacios \& Galdón Delgado, 2007; Mérida Serrano, Torres-Porras \& Alcántara Manzanares, 2017). Al igual que en otras didácticas aplicadas, se va poniendo el énfasis principalmente en procedimientos y contextos, en detrimento de los conceptos (Perales Palacios, 2018). En este sentido, no siempre van de la mano los cambios en la formación inicial de maestros con los de los niveles educativos para los que se prepara a los maestros en formación. Son deseables avances conjuntos que permitan un mejor aprendizaje de las ciencias en todas las etapas educativas, retroalimentándose las innovaciones educativas diseñadas en cada ámbito y coaprendiendo desde la investigación y la aplicación didácticas, en vez de planteamientos independientes no relacionados.

Con el fin de valorar en qué medida existe sinergia entre los planteamientos desarrollados desde las áreas de Didáctica de las Ciencias Experimentales en la formación de maestros y los desarrollados en los centros educativos se han realizado diversas aproximaciones, desde valorar producciones del profesorado en formación en los centros, por ejemplo a través de los Trabajos Fin de Grado implementados en las aulas (Aguirre Pérez, 2018) o el análisis de las observaciones de los procesos de enseñanza-aprendizaje de los centros educativos a través de las percepciones del profesorado en prácticas (Cantó-Doménech, de Pro-Bueno \& Solbes, 2016), si bien la opción más directa de lo que se hace en las aulas proviene de los docentes en activo en esos niveles. Sin embargo, las investigaciones publicadas sobre la implementación en Educación Infantil al respecto resultan escasas (Benarroch, 2010; Cantó-Doménech, de Pro-Bueno \& Solbes, 2016).

En este trabajo, de la Didáctica de las Ciencias Experimentales nos centramos en la Didáctica del Medio Natural, y, más concretamente, en los procesos de enseñanza-aprendizaje llevados a cabo sobre la biodiversidad en la Educación Infantil en los centros asturianos. Este concepto científico, agrupando la variedad de la vida en todos sus niveles, desde genes a ecosistemas, es sumamente empleado en la vida cotidiana (no siempre de una forma correcta) e inserto en última instancia en el núcleo de la Educación Ambiental (tanto desde perspectivas científicas como políticas), recogiéndose su conservación entre los Objetivos de Desarrollo Sostenible.

\section{Investigación de fondo}

La biodiversidad es un concepto que se cree ampliamente conocido, o por lo menos ampliamente comentado en la sociedad y la escuela, así como una necesidad de conservación ambiental. $Y$, sin embargo, algo en estos procesos no acaba de funcionar en los procesos de enseñanza-aprendizaje implementados, cuando nos encontramos cada vez más alejados de la naturaleza (Kesebir \& Kesebir, 2017; Prévot-Julliard, Julliard \& Clayton, 2015; Pyle, 2003), relación que se considera imprescindible para poder conseguir un desarrollo óptimo en la infancia (Corraliza \& Collado, 2011; Torres-Porras et al., 2017).

Aunque se han realizado reflexiones sobre metodologías y conceptos en el aprendizaje de la biodiversidad, suelen estar basados en etapas educativas muy posteriores a la Educación Infantil en la 
educación formal (Bermudez, De Longui, Díaz \& Gavidia Catalán, 2014; Bermudez, De Longhi \& Gavidia, 2016, Bermudez, 2018; García Gómez \& Martínez Bernat, 2010) o experiencias de educación no formal con un público objetivo muy diferente (Marandino \& Díaz Rocha, 2011). Planteamientos generales indican la necesidad de abordar el tema desde actividades cotidianas a partir de acciones concretas, favoreciendo una educación efectiva y transformadora (Gadotti, 2002). La escuela debería permitir integrar el estudio de la biodiversidad al tiempo que potenciar la transición ecológica, invitando, preparando y acompañando al alumnado en estas transformaciones ecosociales (Sauvé, 2017). Estas actuaciones deberían incluir tanto conocimientos directos sobre conceptos, como competencias genéricas de tipo comunicativo y específicas, sobre actitudes y procedimientos (Ruiz Heredia, 2008; Torres et al., 2017).

A tenor de lo indicado por diversos autores, es conveniente que esta Educación Ambiental comience en la primera infancia (Cutter-Mackenzie et al., 2014), integrando variedad de recursos, ya que se ha indicado la importancia de las experiencias científicas (Mérida Serrano et al., 2017), la experimentación directa (Loxley, 2018; Vega, 2012) o las experiencias de conocimiento del entorno en la naturaleza (Wells $\&$ Lekies, 2006). Entre las infraestructuras didácticas del centro escolar que se consideran relacionadas con actividades de aprendizaje de la biodiversidad en Educación Infantil estaría el huerto escolar (Aragón Núñez \& Lorite, 2016; Aragón Núñez, 2017; Ceballos, 2017; Escutia, 2009), el patio con o sin vegetación (Conde Campos \& Pinzolas Torremocha, 2005), el laboratorio escolar (p.e., Vidal López, 2014), un rincón específico en el aula (p.e., Miguélez, Ansedes, Vidal \& Membiela, 2017) y el uso de las Tecnologías de la Información y la Comunicación (TIC) y del Aprendizaje y la Comunicación (TAC), como la pizarra digital y el ordenador conectado a Internet.

Pero la realidad de los centros educativos de Educación Infantil es diversa y heterogénea, y necesario conocer cuál es la situación actual en la que se encuentra el profesorado en activo de la etapa, respecto a la presencia y al uso de las infraestructuras escolares que pudieran ser adecuadas para estos procesos de enseñanza-aprendizaje de la biodiversidad, lo que nos devuelve al punto de que resultan relativamente escasas las experiencias de educación ambiental publicadas en Educación Infantil (y especialmente la relativas a la biodiversidad, véase Cantó Doménech, de Pro Bueno \& Solbes, 2016; Varela-Losada et al., 2016). Y eso, pese a la profusión de modos en las que las maestras de esta etapa exponen de forma heterogénea sus actividades en formatos no académicos (blogs de colegios 0 personales, redes sociales...).

\section{Objetivos / preguntas de investigación}

El objetivo principal de este trabajo es analizar conceptos empleados en el aprendizaje de la biodiversidad en la etapa de Educación Infantil en los centros educativos de Asturias, así como las infraestructuras didácticas disponibles y empleadas en ese proceso de enseñanza-aprendizaje.

Para su consecución se ha dividido en los siguientes objetivos secundarios:

- Averiguar conocimientos existentes acerca de la biodiversidad en docentes en activo de centros educativos de Asturias.

- Recoger cómo se aplica este concepto en Educación Infantil.

- Conocer las diferentes infraestructuras/dotaciones didácticas de cada centro que puedan relacionarse con el aprendizaje de la biodiversidad y si estas son utilizadas en esta etapa educativa. 


\section{Metodología / escenario de investigación}

\subsection{Diseño y aplicación del cuestionario}

El método de investigación utilizado para recopilar la información necesaria para el desarrollo de este trabajo ha sido la encuesta a los docentes en activo de Educación Infantil a través de sus centros educativos. A fin de cuentas, si lo que se quiere investigar es qué intentan las docentes que aprenda el alumnado de Educación Infantil, y con qué metodología y recursos lo intentan, preguntar a dichas docentes en activo parece una buena idea (Cantó Doménech et al., 2016).

Se ha diseñado específicamente para este trabajo un cuestionario en línea mediante la herramienta de Formularios de Google (Google Forms), para facilitar la respuesta en un entorno amigable e independiente del dispositivo del encuestado o su software. Tras diseñar una primera versión del cuestionario, se revisó la adecuación de los ítems a lo que se deseaba valorar, se modificaron las preguntas para su lectura en positivo, se añadieron ítems para facilitar que todo docente interesado en contestar el cuestionario tuviera alguna opción de contestación positiva real con infraestructuras didácticas y se verificó su implementación con respuestas de prueba que reflejaban situaciones reales y que fueron posteriormente eliminadas. El cuestionario final ha incluido en total 19 ítems: cinco destinados a la categorización del centro educativo; uno para comprobar en qué sentido era considerado el concepto de biodiversidad; cinco para explorar cómo se implementa el proceso de enseñanza-aprendizaje de la biodiversidad en la Educación Infantil, en el caso que se realice, y cómo consideran docentes en activo que podría actuar desde esta etapa educativa para fomentar su protección; cuatro ítems para explorar las infraestructuras didácticas disponibles o empleadas en cada centro por el profesorado de Educación Infantil; y tres para indicar percepciones/conocimientos concretos sobre la biodiversidad expresados por los docentes. La exploración pretende recoger la mayor parte de la riqueza de infraestructuras didácticas y metodologías empleadas en los centros educativos asturianos, por lo que se han combinado preguntas de respuestas cerradas con otras de respuestas abiertas y, en todo caso, se ha permitido en las referentes a infraestructuras el relleno de una opción abierta para complementar las propuestas. Finalmente, se ha incluido una pregunta abierta posibilitando la inclusión de comentarios adicionales sobre la enseñanza-aprendizaje de la biodiversidad en Educación Infantil (tabla 1). 
Tabla 1: Esquema del cuestionario diseñado.

\begin{tabular}{ll}
\hline \multicolumn{1}{c}{ Ítems cuestionario } & \multicolumn{1}{c}{ Opciones } \\
\hline Sección categorización centro educativo & \\
\hline Nombre & Pregunta abierta \\
\hline Ubicación & Pregunta abierta (se espera localidad o concejo cuando \\
& posible) \\
\hline Tipo del centro escolar (según tipología Educastur) & Centro de Educación Infantil (CEI) \\
& Centro de Educación Básica (CPEB) \\
& Colegio Rural Agrupado (CRA) \\
& Colegio Público (CP) \\
& Colegio (titularidad privada) (COL) \\
& Escuela de Educación Infantil (EEI) \\
\hline Tamaño de su centro: alumnado & Ninguno de los anteriores \\
\hline Tamaño localidad centro: $n^{\circ}$ personas & Se espera número \\
\hline
\end{tabular}

\section{Sección didáctica de la biodiversidad}

\begin{tabular}{|c|c|}
\hline ¿Cómo definiría el concepto de biodiversidad? & Pregunta abierta \\
\hline $\begin{array}{l}\text { ¿Se trabaja el concepto de biodiversidad en las aulas de } \\
\text { Educación Infantil? }\end{array}$ & Sí/No \\
\hline $\begin{array}{l}\text { Si su respuesta anterior ha sido sí, describa brevemente de } \\
\text { qué modo lo hacen }\end{array}$ & Pregunta abierta \\
\hline ¿Qué actividades enfocadas a este tema se llevan a cabo? & Pregunta abierta \\
\hline $\begin{array}{l}\text { ¿Considera la biodiversidad un aspecto importante a } \\
\text { trabajar en las aulas? }\end{array}$ & Sí/No \\
\hline $\begin{array}{l}\text { ¿Se puede fomentar la protección de la biodiversidad desde } \\
\text { la escuela? ¿cómo? }\end{array}$ & Pregunta abierta \\
\hline ¿Qué tipo de infraestructuras / dotaciones posee el centro? & $\begin{array}{l}\text { Huerto escolar } \\
\text { Laboratorio }\end{array}$ \\
\hline $\begin{array}{l}\text { De las que dispone el centro ¿cuáles se utilizan con } \\
\text { alumnado de Educación Infantil? }\end{array}$ & $\begin{array}{l}\text { Pizarra digital } \\
\text { Patio con zona de vegetación } \\
\text { Patio con (o en inmediación de) charca/río } \\
\text { Otra (se separa como pregunta abierta) }\end{array}$ \\
\hline $\begin{array}{l}\text { ¿Hay alguna especie en peligro de extinción en la zona en la } \\
\text { que se encuentra? ¿cuál(es)? }\end{array}$ & Pregunta abierta \\
\hline $\begin{array}{l}\text { ¿Qué problemas pueden surgir a raíz de la pérdida de la } \\
\text { biodiversidad? }\end{array}$ & $\begin{array}{l}\text { Extinción de especies } \\
\text { Pérdida de la diversidad funcional } \\
\text { Impacto sobre los ecosistemas } \\
\text { Impactos económicos para el ser humano } \\
\text { Impactos culturales para el ser humano }\end{array}$ \\
\hline $\begin{array}{l}\text { Considera que las causas principales que están ocasionando } \\
\text { la pérdida de la biodiversidad son: }\end{array}$ & $\begin{array}{l}\text { Calentamiento global } \\
\text { Falta de reproducción de las especies } \\
\text { Ausencia de alimentos } \\
\text { Ausencia de agua } \\
\text { Presencia de especies invasoras } \\
\text { Destrucción del hábitat } \\
\text { Caza/pesca/recolección } \\
\text { Contaminación ambiental }\end{array}$ \\
\hline $\begin{array}{l}\text { Algún otro comentario sobre la enseñanza-aprendizaje } \\
\text { biodiversidad en Educación Infantil }\end{array}$ & Pregunta abierta \\
\hline
\end{tabular}


Dicho cuestionario se ha enviado por correo electrónico en tres ocasiones (en abril, principios y finales de mayo 2018) a los 385 centros en los que se imparte Primer o Segundo Ciclo de Educación Infantil en Asturias, de acuerdo con la base de datos de la Consejería de Educación del Gobierno del Principado de Asturias (Educastur, 2018; https://www.educastur.es). En el segundo y tercer envío no se incluyeron los centros que ya habían respondido (aunque fuera para contestar que no iban a realizar la encuesta), para limitar las molestias para el personal docente.

\subsection{Caracterización de la muestra obtenida}

Se han obtenido 58 cuestionarios respondidos, los que representa un $15 \%$ del total de centros educativos de Asturias que incluyen la etapa de Educación Infantil en primer o segundo ciclo. Este nivel de respuesta no ha sido homogéneo entre tipos de centros educativos, siendo bastante inferior entre centros de Primer Ciclo de Educación Infantil (11\% de las EEI, $8 \%$ de los CEI) y muy superior entre las tipologías menos habituales (19\% de los CRA y $22 \%$ de los CPEB). El resto de los centros educativos con Segundo Ciclo de Educación Infantil, los más numerosos, respondieron en un porcentaje muy similar (14\% los de titularidad pública, $15 \%$ los de privada).

Casi la mitad (47\%) de la muestra así obtenida está formada por Colegios Públicos con Segundo Ciclo de Educación Infantil. Los colegios de titularidad privada del Segundo Ciclo representan un 16\%, mismo porcentaje que las Escuelas de Educación Infantil de Primer Ciclo, representando un porcentaje inferior otras tipologías (Figura 1).

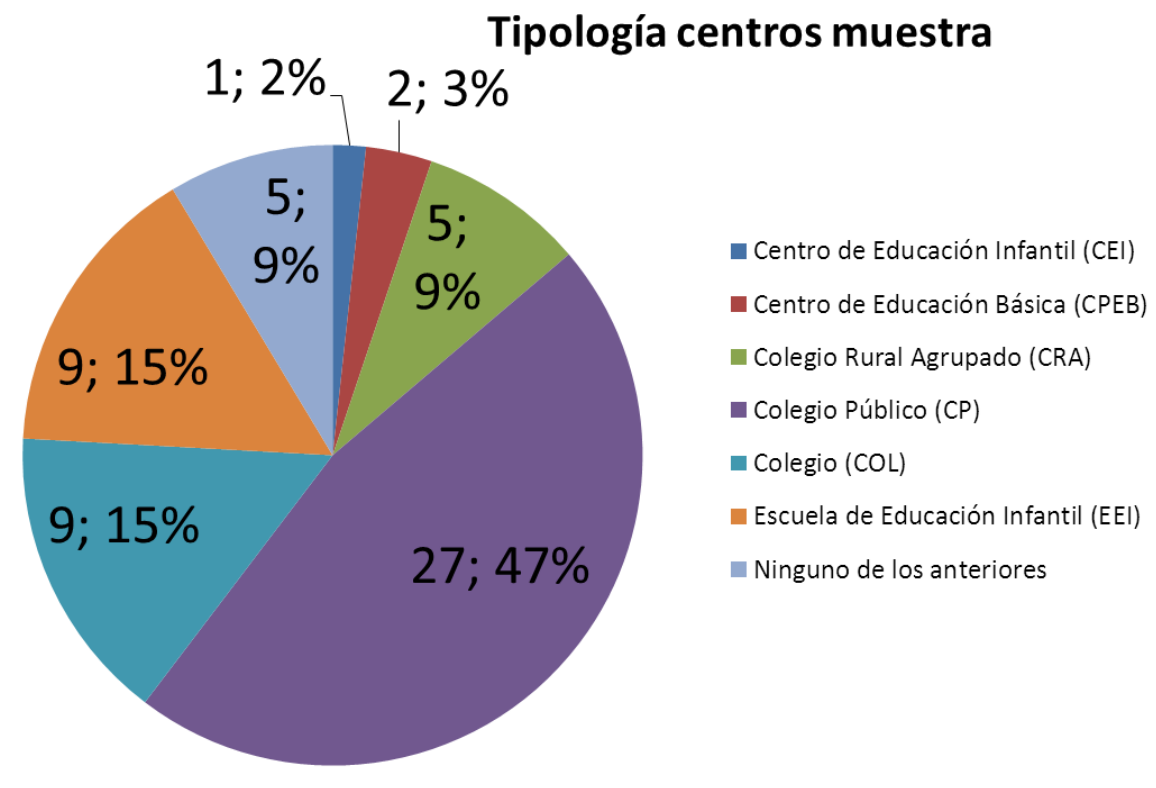

Figura 1. Caracterización de los centros presentes en la muestra según su tipología ( $n=58)$.

El tamaño de los centros educativos que han contestado varía entre algo menos de 20 alumnos y casi 2000 , si bien el $60 \%$ de los centros se encontraban por debajo de los 200 alumnos y un tercio entre los 300 y los 1000, con diferencias entre tipologías (Figura 2). 


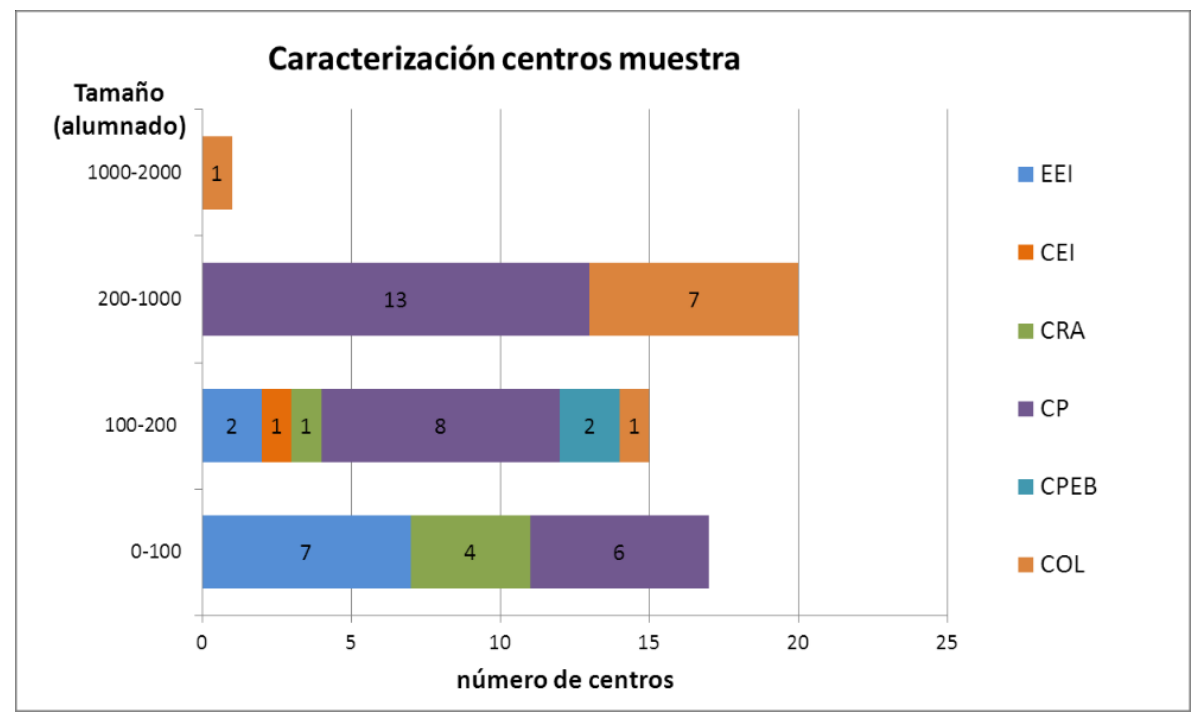

Figura 2. Caracterización de los centros que han contestado según tamaño y tipología.

La mitad (52\%) de los centros educativos que respondieron se encuentran en localidades de más de 10000 habitantes (de hecho, el 22\% de los centros pertenecen a la capital Oviedo, y el $9 \%$ a la ciudad con mayor población de Asturias, Gijón), estando igualmente representados los pertenecientes a localidades de menos de 1000 habitantes y entre 1000 y 10000.

\section{Resultados}

\subsection{Conocimientos sobre biodiversidad}

La primera pregunta que nos encontramos está relacionada con el tema central de la encuesta, es decir el concepto de Biodiversidad. Para averiguar qué concepto manejaban los docentes de Educación Infantil se les ha preguntado cómo lo definirían, obteniendo la respuesta de 56 docentes. El concepto de biodiversidad está plenamente integrado en nuestra cultura, y en general los docentes manejan el mismo significado, aunque puedan expresarlo de formas distintas. La biodiversidad está explícitamente asociada al concepto de variedad en un tercio de las respuestas (32\%), y al más literal de diversidad en casi otro cuarto (23\%). En la mayor parte de los centros los docentes definen la biodiversidad como la variedad de especies que viven en un espacio determinado, si bien el $9 \%$ indican el conjunto del planeta como ese territorio, haciendo referencia por tanto a la biodiversidad de La Tierra en su conjunto. En algunos casos especifican que son especies animales y vegetales (29\%), en otros indican que son el conjunto de los seres vivos (20\%), de las formas de vida o de la vida en sí misma (18\%), pero manteniendo el significado del concepto en su definición.

No obstante, también se han localizado concepciones alternativas para el término biodiversidad, que no se corresponderían con la definición de Edward O. Wilson ni la de los manuales de Ecología al uso. Así, el $4 \%$ de los docentes indican que la biodiversidad es el estudio de las diferentes especies animales y vegetales, otro $4 \%$ considera que hace referencia a su conservación, otro $4 \%$ considera que es la convivencia de las especies (para un $2 \%$ en convivencia armoniosa) y otro $2 \%$ considera equivalentes los conceptos de biodiversidad y entorno natural. Finalmente, una respuesta muestra confusión con el tema del formulario, al asociar el concepto a la diversidad del alumnado.

Una vez definido el concepto de biodiversidad, se buscó comprobar si la identificaban como parte de las cuestiones que se trabajaban en Educación Infantil, contestado afirmativamente por el $86 \%$ de los 
docentes que respondieron. No obstante, todos los centros consideran que es un aspecto importante que trabajar en las aulas.

\section{2 ¿Cómo se trabaja la biodiversidad en Educación Infantil?}

A aquellos que han contestado que lo trabajan en Educación Infantil, se les ha pedido indicar de qué modo se hace. Un $14 \%$ no indican la metodología concreta que emplean, sino que exponen el aprendizaje sobre flora y fauna o ciertos ecosistemas de una forma general. En cambio, el $44 \%$ de los centros afirma emplear "proyectos" para el trabajar la biodiversidad, si bien no se ha analizado el concepto de "proyecto" considerado por cada centro, y las repuestas proporcionadas parecen mostrar una cierta heterogeneidad de significados para docentes en activo. El $5 \%$ indican que realizan proyectos o unidades didácticas concretas referidas a animales (ya sea en general, a un grupo de ellos o a una especie en concreto) y al medio ambiente, mientras que otro $5 \%$ indican que lo integran en otras (o en todas) las unidades didácticas que trabajan a lo largo del curso. Así, el $4 \%$ afirman consideran como uno de los centros de interés dentro de cada proyectos o unidad didáctica a los animales, mientras que el $2 \%$ incluye también a las plantas como centro de interés. La tercera parte (34\%) asocia el aprendizaje de la biodiversidad con aquella que se encuentra en su entorno más próximo (desde trabajos y observaciones en el recinto del colegio - patio y/o huerto- hasta las cercanías al centro). Un 10\% especifica el uso del huerto escolar para el aprendizaje de la biodiversidad, mientras que sólo un $7 \%$ de los centros escolares afirma realizar salidas didácticas para su aprendizaje, correspondiéndose con un CRA y tres CP con menos de 300 alumnos cada uno. El empleo de cuentos o libros infantiles para esta cuestión es indicado únicamente por un $3 \%$.

Algunas de las respuestas son sumamente esclarecedoras en cuanto a los conceptos que se aprenden o la metodología que se sigue en el centro educativo al tratar este tema, por lo que las transcribo a continuación

- Se trabaja desde los ecosistemas, desde la diversidad genética pero también cultural. Se trabajan también todas las conexiones y relaciones entre especies y entre especies y entorno.

- El colegio se encuentra en un entorno privilegiado, con $100 \mathrm{~m}^{2}$ de naturaleza cuidada, con 150 especies de árboles y arbustos catalogados en el jardín botánico.

- Se trabaja de distintas formas. Ya sea a través de comentarios en asamblea en las que los niños cuentan experiencias, en los libros que se llevan a casa o como contenido propiamente dicho a través de actividades de fichas o proyectos.

- Acercamos a nuestro alumnado a su entorno inmediato, a través de distintas experiencias y proyectos experimentan en ocasiones, verbalizan en otras y disfrutan en todas del entorno que les rodea, animales, plantas, climatología, sabores... Partimos del entorno inmediato y de sus cambios a lo largo del año. Después pasamos a otros entornos que no viven cotidianamente: selva, mares, continentes... También intentamos tener algún animal en la escuela con lo que fomentamos el cuidado de seres vivos, ahora tenemos gusanos de seda.

- Tipos de árboles y paisajes, climas, proyecto la vuelta al mundo.

- Desde la necesidad de conocer en qué modo las personas nos beneficiamos de la misma y de qué manera influimos nosotras, las personas, en la misma.

- A través de la sensibilización y la autoconstrucción de conocimiento sobre el medio que nos rodea, la implicación y participación del alumnado en la defensa y protección del entorno cercano en todos sus comportamientos cotidianos. 
- A través de educación en valores, de manera transversal.

\subsection{Tipo de actividades implementadas}

La mayor parte de los centros afirman trabajarlo mediante salidas didácticas, la realización de proyectos, haciendo uso del huerto escolar, y mediante el reciclaje. También hay centros que trabajan los animales y plantas de los distintos entornos, ven documentales y hablan sobre ellos, lo trabajan desde la perspectiva del cuidando el medio ambiente, mediante la colaboración familiar, el trabajo globalizado, realizando experimentos o investigaciones. Otros describen actividades más concretas como la plantación de semillas o la existencia de gusanos de seda en el aula.

Algunas de las respuestas que destacaría son las siguientes:

- Cambio climático. Contaminación. Relación del hombre con el medio que lo rodea. Actitudes responsables. Consumo responsable. Conocemos algunas especies del planeta y también pequeños ecosistemas como una charca. Visita al jardín botánico. Este año se trabaja también desde Célula Europa.

- Dentro de las programaciones didácticas, existen actividades destinadas a la integración de los niños con el entorno. Paseos por los alrededores, para ver los caballos de la hípica vecina, jugar en el riachuelo cercano y sobre todo aprender a valorar desde muy pequeños a todos los seres vivos que nos rodean. En infantil hay diseñadas unidades de indagación específicamente diseñadas para trabajar la biodiversidad y este trabajo se profundiza en primaria.

- Este trimestre, concretamente, estamos trabajando el proyecto de las abejas. Con ello los niños conocen las características y formas de vida de este ser vivo junto con la importancia que tienen para los humanos.

- Cada programación mensual dedicamos un apartado al medio ambiente y en primavera y verano, por la climatología especialmente. Participamos en un eco huerto, se planta, se cuida y se observa la evolución de las plantas. Tenemos algún animal en la escuela que cuidamos todo el curso: peces, tortugas, gusanos de seda. Y ven su desarrollo. Elaboración de materiales de reciclaje, etc...

- En el caso de nuestro centro trabajamos con una metodología de ABP, el mundo de los animales, un proyecto de huerto escolar ecológico, un proyecto sobre el reciclado de los materiales de desecho, un proyecto de alimentación saludable.

La mayor parte de los centros coincide con las respuestas anteriores, con la diferencia que algunos han tratado de ser más explícitos.

Otra de las preguntas que se les ha planteado es si se puede fomentar la protección de la Biodiversidad desde la escuela, y de qué forma. Las respuestas más frecuentes han sido el cuidado y respeto de la naturaleza, el conocimiento de las especies, explicar la importancia que tiene, dando ejemplo, reciclando o visitando ecosistemas de nuestro alrededor.

Sin embargo, también se han registrado otras respuestas diferentes a las anteriores, como, por ejemplo:

- Actitud y consumo responsable. Cuidado y respeto a las plantas y animales de nuestro entorno más cercano que se puede extrapolar a entornos más abiertos o lejanos. Visitas al Eco huerto. Visitas al zoo, acuarios. Visitas a centros de rescate de animales. 
- Desde la transmisión de los valores y la sensibilidad por la naturaleza. La toma de conciencia de los niños sobre la importancia de cuidar y proteger el entorno. El papel responsable que tenemos en el mantenimiento de un entorno sano.

- Enseñándoles a valorarla, cuidarla y respetarla. En infantil es muy importante actuar como modelos y en nuestro día a día llevar a cabo medidas como cuidar las plantas de nuestro patio o del aula y aprender a no molestar a los animales que vienen en ellas. También darle importancia al reciclaje o la reutilización de materiales de aula. Los más pequeños tienden a deshacerse de las cosas rápidamente. En nuestra aula las cosas se usan varias veces para distintos fines normalmente relacionados con manualidades.

- Con un taller sobre la diferencia entre abejas y avispas asiáticas, otro sobre la contaminación de los ríos y cómo afecta a la vegetación y fauna del río.

\subsection{Infraestructuras didácticas disponibles y utilizadas}

Únicamente un $14 \%$ de los centros dispone solo de una de las infraestructuras/dotaciones por las que se preguntaba (bien la pizarra digital, bien el huerto escolar o el rincón específicamente diseñado para este fin). La cuarta parte de los centros disponía de dos de estos recursos didácticos, otro cuarto de tres, un $18 \%$ cuatro y solo un $7 \%$ cinco. El elemento presente en casi todos los centros es la Pizarra Digital (79\%), dos tercios poseen un huerto escolar (72\% si les sumamos los que emplean como sustituto huertos verticales o de mesa, o realizan visitas a huertos cercanos, fuera del colegio), el 60\% (al menos un) patio con (al menos una) zona con vegetación, un poco menos de la mitad un laboratorio (44\%), y un $12 \%$ el patio con (o inmediación) charca o río (Figura 3). Otras infraestructuras didácticas consideradas por los maestros han sido la compostadora, una pomarada (campo de manzanos), un parque cercano, un rincón específico para el estudio de la biodiversidad (con terrarios, pequeñas plantaciones y experimentos) y un arenero (si bien no se han indicado actividades concretas en este último).

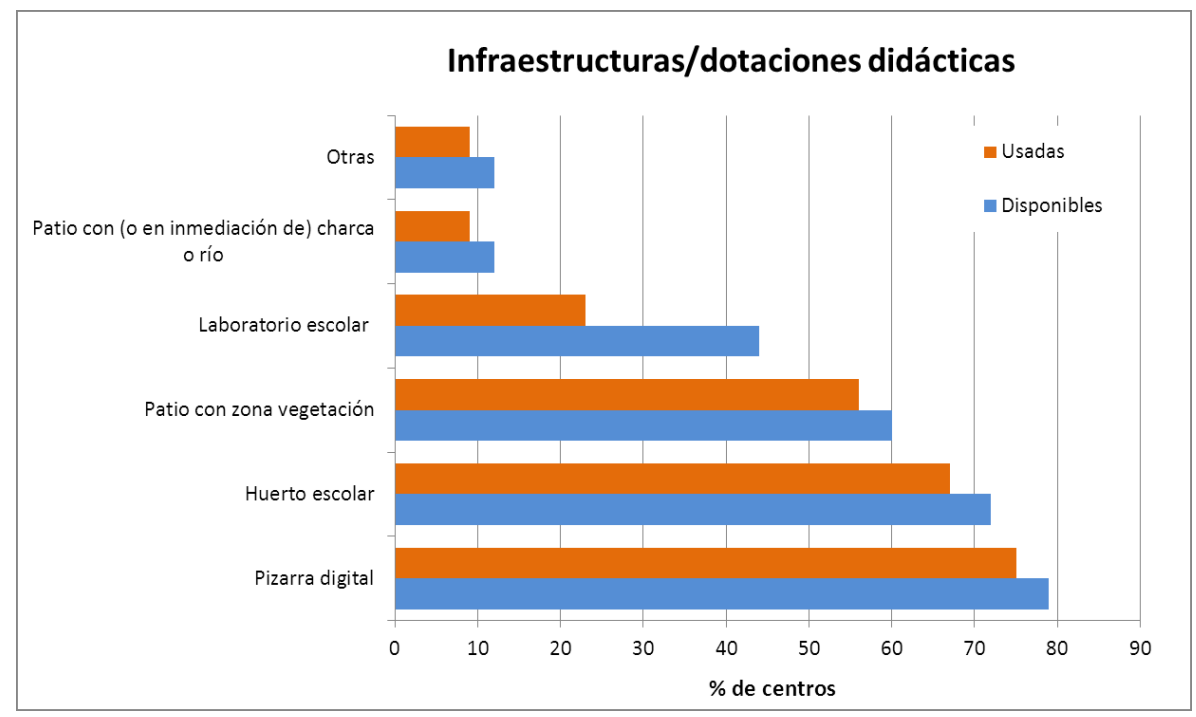

Figura 3. Infraestructuras o dotaciones didácticas disponibles y utilizadas en los centros educativos para el aprendizaje de la biodiversidad en Educación Infantil $(n=55)$.

Se observan algunos planteamientos generales diferentes según tipología de centros. Así, únicamente el $11 \%$ de los colegios de titularidad privada que han respondido disponen de un huerto escolar, mientras que las tres cuartas partes de los colegios públicos lo tienen, de forma similar a las Escuelas de Educación Infantil, siendo más elevados los porcentajes en el resto de tipologías. En el caso del patio 
con zona con vegetación, los porcentajes disminuyen al $66 \%$ en los colegios públicos, $44 \%$ en las Escuelas de Educación Infantil y no se dispone en el único CEI que contestó, manteniéndose en el resto como el recurso anterior. El laboratorio presenta el patrón contrario: todos los colegios de titularidad privada afirman poseer uno, al igual que los Colegios Públicos de Enseñanzas Básicas, porcentaje que baja al $41 \%$ en los colegios públicos y a ninguno en los Colegios Rurales Agrupados (los centros de primer ciclo no disponen ni necesitan laboratorio, como es esperado).

El porcentaje de centros que, disponiendo de estas infraestructuras, las utilizan en Educación Infantil, es muy elevado, rondando o superior al $90 \%$ salvo en el caso del huerto, con un empleo un poco inferior (83\%) y el laboratorio escolar, ese sí muy poco empleado en Educación Infantil (solo el $23 \%$ de los centros que dicen disponer de él lo utilizan en esta etapa educativa).

Al hablar sobre biodiversidad, se ha considerado apropiado preguntar si en sus respectivas zonas hay algún tipo de especie en peligro de extinción, mostrando que la gran mayoría de los centros desconocen dicho dato, otros en cambio niegan su existencia. Entre los que han contestado de forma afirmativa, se observa el predominio de animales como el oso pardo, seguido del urogallo, águila, buitre, salmón o el cormorán [moñudo] (Figura 4). Teniendo en cuenta las respuestas, en muchos casos la zona a la que parecen referirse es la comunidad autónoma, en otros podría ser el municipio. Los ejemplos de plantas amenazadas son más raros: el acebo y plantas dunares. En un caso se indican otras especies que no se consideran catalogadas, pero sí con poblaciones disminuidas en el concejo que se pueden tratar en el aula (murciélagos, cucos, golondrinas, ranas, codornices, lobos, castaños...) (no obstante, algunas de ellas sí que estarían también catalogadas).

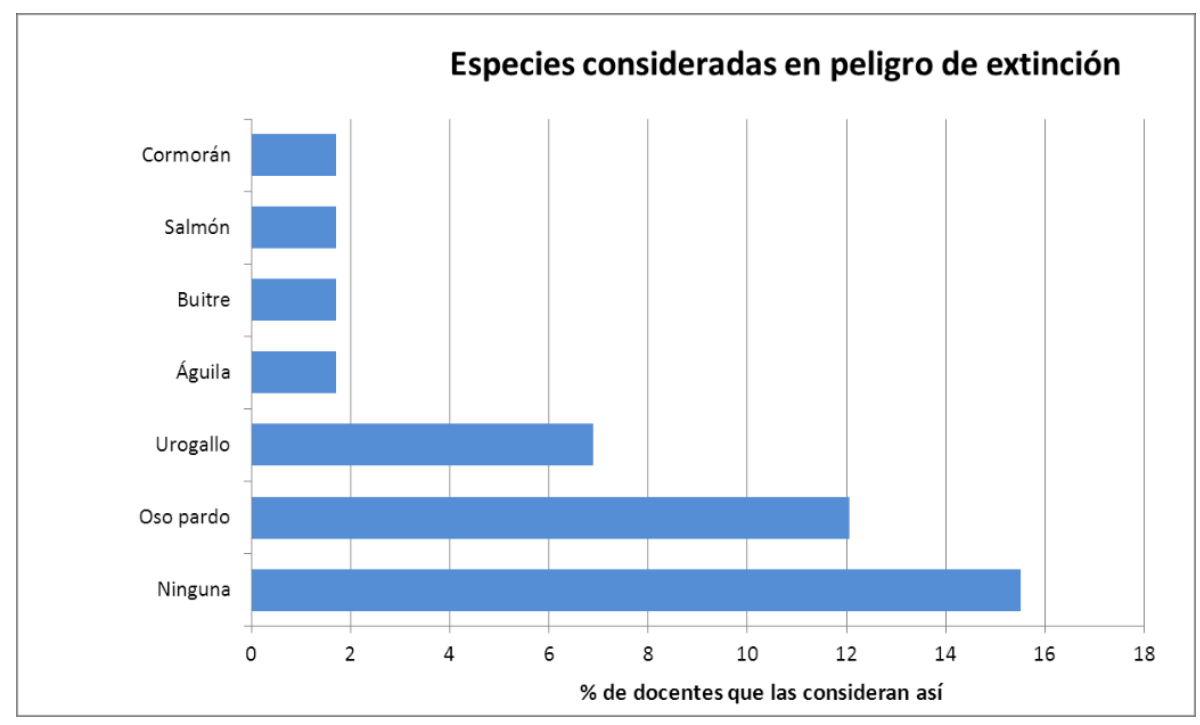

Figura 4. Especies consideradas por los docentes de Ed. Infantil en peligro de extinción en las cercanías de los centros $(n=33)$.

Los docentes en activo seleccionan principalmente como causas de la pérdida de la biodiversidad el calentamiento global, la contaminación ambiental y la destrucción del hábitat (Figura 5). En cuanto a los problemas que puede generar esta destrucción de la biodiversidad, los que han sido percibidos como más importantes han sido la extinción de especies y su impacto sobre los ecosistemas (Figura 6). 


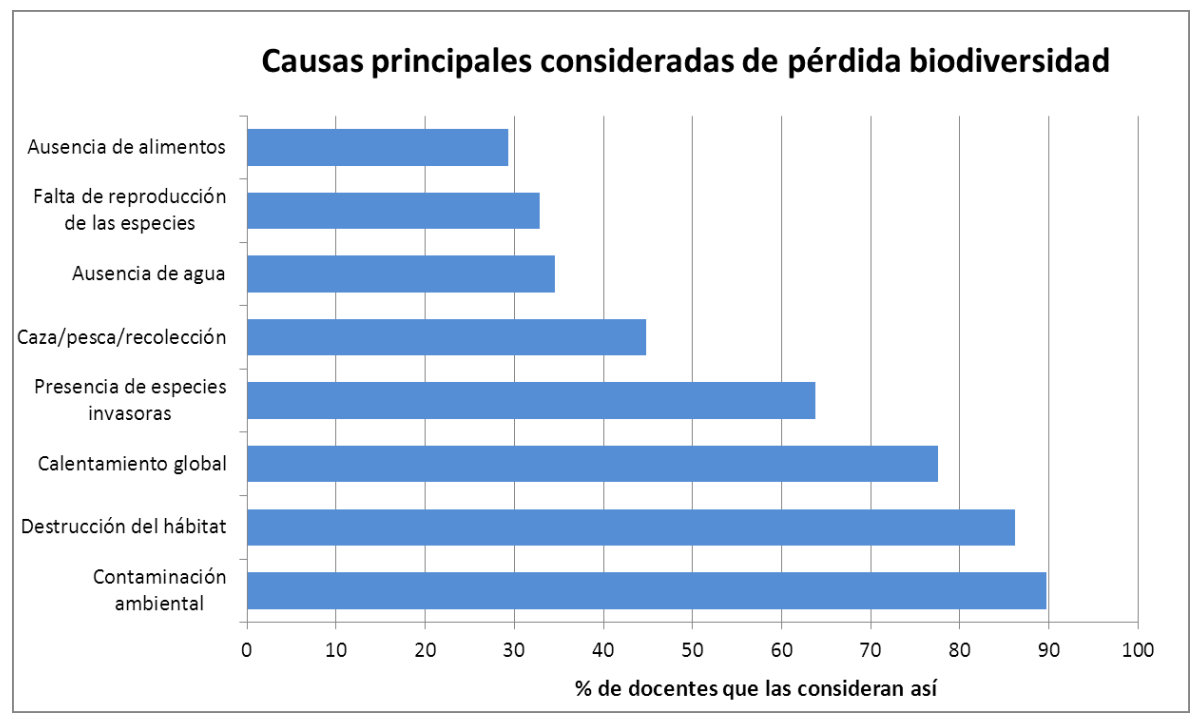

Figura 5. Percepciones de los docentes en activo de Educación Infantil sobre las causas principales que están ocasionando la pérdida de la biodiversidad ( $n=58$, cada docente podía elegir todas aquellas que considerara causas principales).

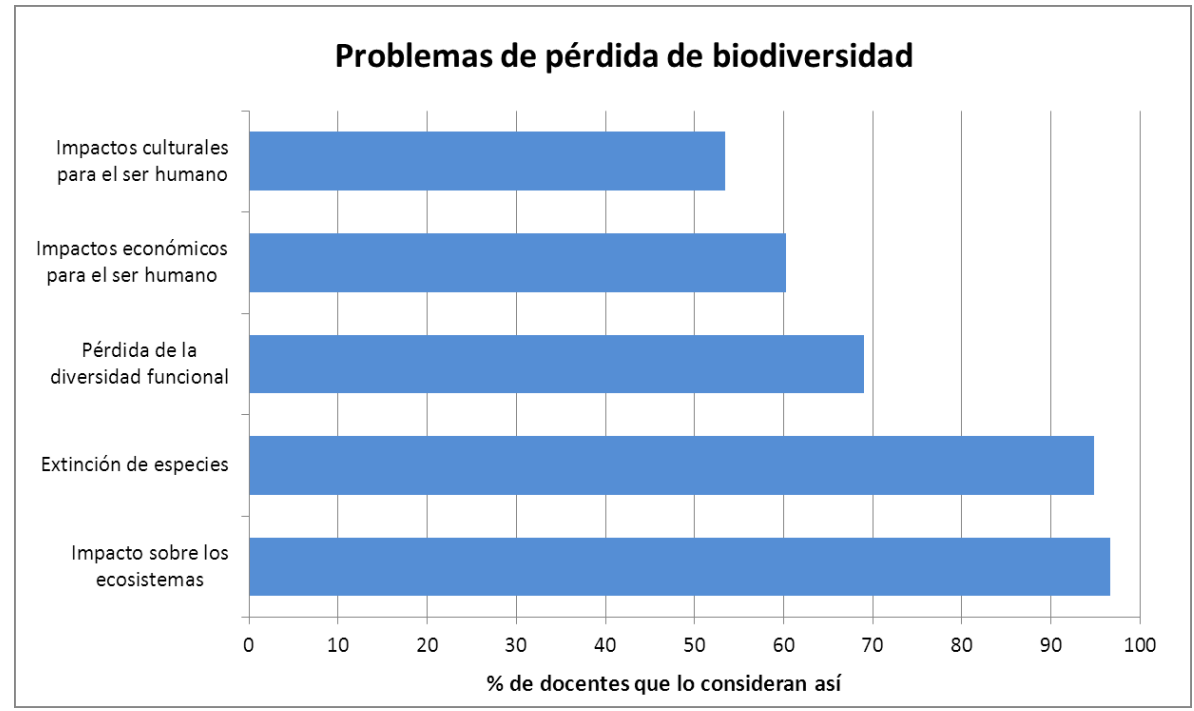

Figura 6. Percepciones de los docentes en activo de Educación Infantil sobre los problemas que puede originar la pérdida de la biodiversidad $(n=58)$

\section{Discusión}

El concepto de biodiversidad está plenamente integrado en nuestra cultura, y en general los docentes manejan un significado similar, aunque puedan expresarlo de formas distintas. Diversidad y variedad están íntimamente ligadas a su definición para gran parte de los docentes, en gran parte identificados con la diversidad de especies de una zona determinada. Definiciones más o menos académicas o de aceptación más general, como la del Convenio de Naciones Unidas sobre Conservación y Uso Sostenible de la Diversidad Biológica (https://www.cbd.int/), inciden en que la variabilidad que compone la biodiversidad incluye más que la diversidad de especies, dando cuenta también de la variabilidad dentro de las especies y de la variabilidad de los ecosistemas y de los complejos ecológicos de los que forman parte. Esta ampliación del concepto de biodiversidad es tenida en cuenta solo por un porcentaje menor 
del profesorado de infantil en activo (por lo menos conscientemente, según las respuestas al cuestionario). En este contexto, los docentes en ejercicio poseen información y conocimientos, en mayor o menor medida, pero es posible que fuera necesario refrescarlos en aquellos casos que más se alejan del concepto de biodiversidad. Confusiones de la biodiversidad con su estudio o con su conservación (o con la sensibilización ambiental) alcanzan el $8 \%$ de las respuestas, tal vez debido a que se abordan de forma conjunta con este concepto. Otras concepciones alternativas pueden considerarse más alejadas, como el que la biodiversidad implica convivencia armoniosa, por ejemplo. La pregunta sobre especies amenazadas tenía por objeto valorar en qué medida ese conocimiento de la biodiversidad, aplicado a una cuestión local y singular, estaba interiorizado entre los docentes. Los docentes consideran importante sensibilizar al alumnado sobre la biodiversidad y las especies amenazadas, pero luego muestran respuestas relativamente escasas sobre su posible presencia en el entorno próximo (o regional, dependiendo de las respuestas obtenidas). El Listado de Especies Silvestres de Protección Especial y Catálogo Español de Especies Amenazadas incluye unas 900 especies, mientras que el Catálogo Regional de Especies Amenazadas de Asturias incluye poco más de 80 (en ambos casos considerando animales y plantas) y la mayoría de los docentes ha respondido con alguna especie efectivamente amenazada, pero estas han sido en general las más llamativas, casi siempre animales emblemáticos en Asturias (oso pardo, urogallo, águila...), escasamente nombradas algunas plantas y ningún invertebrado. Sí que resulta positiva la inclusión entre las respuestas de grupos, efectivamente amenazados, que no suelen considerarse como tales o no tratarse de esa manera en Educación Infantil (p.ej., murciélagos, salmón...).

Uno de los resultados más sorprendentes ha sido el $13,8 \%$ que considera que no trabaja la biodiversidad en las aulas de Educación Infantil. Esto puede estar relacionado con lo analizado en centros educativos de Valencia, donde maestras en formación indicaban que en las aulas de Educación Infantil donde realizaban sus prácticas se trabajaban conceptos de ciencia más como una incorporación inconsciente en las clases que intencionada (Cantó Doménech et al., 2016). Se pueden observar paralelismos con alguna de las respuestas recibidas, en la que la docente indicaba que no se trabajaba la biodiversidad como tal, pero sí especies animales y vegetales. En todo caso, hay que tener en cuenta que los animales son una de las temáticas científicas más tratadas en Educación Infantil, o por lo menos así es percibido por los maestros en formación (de Pro Chereguini, de Pro Bueno \& Rodríguez Moreno, 2018).

Además, cabe destacar, tal y como indican en otras de las preguntas de la encuesta, que todos son conscientes de la gran importancia que tiene la biodiversidad y que se puede fomentar su protección desde la escuela. Además de aquellos que incluyen la sensibilización ambiental o de conservación de la biodiversidad directamente dentro de su concepto de biodiversidad, docentes en activo de Asturias realizan actividades de reciclaje, inculcan hábitos basados en el cuidado y respeto de la naturaleza, imparten conocimientos sobre especies, realizan salidas didácticas...

El modo de trabajar este concepto y las actividades llevadas a cabo en las aulas, son muy diversas tal y como se ha visto en los datos aportados. La omnipresente pizarra digital es el recurso más empleado para explicar cuestiones relativas a la biodiversidad en Educación Infantil, al igual que lo observado en centros valencianos para el conjunto del aprendizaje en ciencias experimentales (Cantó Doménech et al., 2016). No obstante, poco se indica en las respuestas de la metodología seguida con esta dotación, más allá de la utilización para mostrar información o vídeos sobre seres vivos. La introducción de cuestiones relacionadas con la biodiversidad en los proyectos o unidades didácticas desarrolladas en el aula de infantil, o su integración de forma general en los procesos de aprendizaje, son mayoritarios, siendo más escasos los planteamientos en los que se realizan proyectos específicos para ello. Estas cuestiones también tienen cierto paralelismo con lo observado en Valencia: inclusión en casi todos los casos de contenidos sobre los seres vivos ( $89 \%$, pero el que no respondieran afirmativamente todos los docentes es posible que se deba a diferencias con la forma de enfocar la respuesta), aunque con tratamiento específico menor. 
La presencia del huerto escolar y su empleo en Educación Infantil resulta amplia en el caso de Asturias (67\%), diez puntos más alto que en el caso valenciano (57\%). Esta amplia utilización puede estar relacionada con la popularización del uso de esta infraestructura didáctica en los últimos años (Escutia, 2009; Romón Salinas, 2014; Eugenio Gozalbo \& Aragón Núñez, 2016, y referencias allí incluidas). Y, no obstante, hay algunas cuestiones que llaman profundamente la atención de las respuestas obtenidas. La primera, que el porcentaje de centros que dicen emplear el huerto escolar para el aprendizaje de la biodiversidad es mucho más escaso (10\%) que los que dicen utilizarlo en esta etapa, por lo que claramente es un recurso infrautilizado en Educación Infantil, al que se le podría sacar más partido con un diseño de las actividades a realizar que tuviera en cuenta la biodiversidad. La segunda cuestión que llama la atención es la importante diferencia entre tipologías de centros en cuanto a la presencia y uso del huerto escolar. El porcentaje de los colegios de titularidad privada que han contestado a la encuesta que presenta huerto escolar es muy inferior al de los colegios de titularidad pública, las escuelas de primer ciclo y el resto de las tipologías.

Las salidas didácticas para el estudio de la biodiversidad son menos utilizadas de lo esperable, especialmente si lo comparamos con los resultados valencianos, donde casi el $80 \%$ de los centros afirmaban realizar salidas a parajes naturales (Cantó Doménech et al., 2016). Las discrepancias podrían deberse a que efectivamente los centros asturianos que han contestado a la encuesta realizan menos salidas, o a que sí que las realizan, pero no las asocian a aprendizajes relacionados con la biodiversidad, de forma similar a lo que pasaba con la utilización del huerto escolar.

El patio con (al menos una) zona con vegetación es también una infraestructura muy utilizada por las docentes de Educación Infantil en los centros asturianos, aparentemente donde se llevan a cabo experiencias del alumnado con la biodiversidad de su entorno cercano. Y es que la mejora de los patios desde un punto de vista ambiental, con la inclusión de zonas verdes y/o árboles, permite convertirlos en espacios para el aprendizaje de la biodiversidad (Conde Campos \& Pinzolas Torremocha, 2005).

El laboratorio escolar es de las infraestructuras menos utilizadas en Educación Infantil, lo que es normal porque la mayor parte de las actividades experimentales que se plantean en esta etapa podrían realizarse también en el aula, sin ser por tanto realmente necesario el laboratorio (como infraestructura) en ninguno de los dos ciclos de esta etapa (ver ejemplos de actividades en Vega, 2006, 2012). De hecho, ninguno de los centros de primer ciclo dispone de uno. En el caso de los de segundo ciclo, se indica una mayor presencia y un mayor uso en Educación Infantil en los colegios de titularidad privada frente a los de titularidad pública (33 frente a 7\%).

Otras infraestructuras menos comunes, detectadas cada una en un solo centro, y que aportan una gran riqueza para el estudio de la biodiversidad con un correcto aprovechamiento, pueden ser una pomarada (campo con manzanos) y una zona arbolada de especies autóctonas variadas (el "bosque escolar"). Su integración en los procesos de enseñanza-aprendizaje es variada, pero ambos elementos son valorados como señas importantes en sus centros, con actividades específicas de aprendizaje de la biodiversidad, el medio natural y las ciencias en el segundo ciclo de Educación Infantil.

Aquellos centros que han manifestado la realización de más actividades, con mayor abanico de metodologías y más dirigidas al aprendizaje de la biodiversidad, han sido aquellos que disponían de más infraestructuras didácticas relacionadas. En especial, aquellos que disponían de patios naturalizados (al menos parcialmente), con zonas con vegetación y huertos escolares. También aquellos que aprovechaban otras formas de acceso a la biodiversidad saliendo a visitar equipamientos didácticos externas (como centros de interpretación, aunque sin haber manifestado asistencia a aulas específicas de naturaleza como lo recogido en Sanz Alonso, Sistiaga Poveda, Urkidi Elorrieta, Andoño Erdozain \& Orbegozo Rezola, 2018). Para un óptimo desarrollo de los procesos de enseñanza-aprendizaje la 
biodiversidad es importante que los centros dispongan de las infraestructuras necesarias, y que las empleen en esta etapa educativa.

\section{Conclusiones}

- El concepto de biodiversidad es generalmente manejado con un significado similar entre docentes en activo de Educación Infantil, incidiendo en su relación con diversidad y variedad/variabilidad, si bien se dan también interpretaciones más restringidas de lo que se interpreta por variedad total de la vida y se han detectado otras concepciones alternativas.

- El concepto de especie amenazada es empleado de forma mucho más restringida por la mayoría de los docentes de Educación Infantil que lo aplicado en Biología de la Conservación y en la legislación ambiental, indicando las respuestas que se debe mejorar la información que reciben/han recibido a este respecto.

- Los $13,8 \%$ docentes en activo indican que no trabajan la biodiversidad en las aulas de Educación Infantil, mostrando más bien diferencias conceptuales sobre lo que representa la biodiversidad o su aprendizaje que el hecho de que realmente no se haga en las aulas.

- Todos los docentes consideran que la biodiversidad reviste gran importancia y que se puede fomentar su protección desde la escuela. Suelen incluir la sensibilización ambiental o actividades relacionadas en sus programaciones didácticas.

- Las dotaciones/infraestructuras didácticas más utilizadas en Educación Infantil para el aprendizaje de la biodiversidad han sido la pizarra digital interactiva, huerto escolar y patio con vegetación. Se detectan recursos singulares de gran interés, como un "bosque escolar" y una pomarada.

- Las salidas didácticas para el aprendizaje de la biodiversidad y el laboratorio escolar son menos empleados (en este aprendizaje o en general en la Educación Infantil).

\section{Referencias bibliográficas}

Aguirre Pérez, C. (2018). Medio Ambiente y Sostenibilidad en los Trabajos Fin de grado de los futuros maestros de Infantil y Primaria. En: C. Martínez Losada \& S. García Barros (eds.) 28 Encuentros de Didáctica de las Ciencias Experimentales, (pp. 511-516). A Coruña: Universidade da Coruña.

Aragón Núñez, L. (2017). El huerto ecológico: Un recurso innovador para contribuir a las competencias para el desarrollo sostenible en la formación inicial de maestros/as. En S. Pérez-Aldeguer, G. CastellanoPérez, y A. Pina-Calafi (Coords.), Propuestas de Innovación Educativa en la Sociedad de la Información (pp. 136-146). Eindhoven, NL: Adaya Press.

Aragón Nuñez, L. \& Lorite, I.M.C. (2016). Del Huerto Ecológico Universitario al aula de infantil: experiencias educativas en torno a problemas ambientales en la etapa de Infantil. Educación preescolar e infantil, 2(1), 45-46. 
Benarroch, A. (2010). La investigación en Didáctica de las Ciencias Experimentales en las etapas educativas de Infantil y Primaria. En: Abril, A.M. \& Quesada, A. (eds). XXIV Encuentros de Didáctica de las Ciencias Experimentales (pp. 32-52). Baeza: Ser. Pub. Univ. Jaén

Bermudez G.M.A. (2018). ¿Cómo tratan los libros de texto españoles la pérdida de la biodiversidad? Un estudio cuali-cuantitativo sobre el nivel de complejidad y el efecto de la editorial y año de publicación.. Revista Eureka sobre Enseñanza y Divulgación de las Ciencias, 15(1), 1102. Doi: 10.25267/Rev_Eureka_ensen_divulg_cienc.2018.v15.i1.1102

Bermudez, G.M.A., De Longhi, A.L., Díaz, S. \& Gavidia, V. (2014). La transposición del concepto de diversidad biológica. Un estudio sobre los libros de texto de la educación secundaria española. Enseñanza de las Ciencias, 32(3), 285-302. Doi: 10.5565/rev/ensciencias.1129

Bermudez G.M.A., De Longhi A.L. \& Gavidia V. (2016). El tratamiento de los bienes y servicios que aporta la biodiversidad en manuales de la educación secundaria española: un estudio epistemológico. Revista Eureka sobre Enseñanza y Divulgación de las Ciencias, 13(3), 527-543.

Cantó Doménech, J., de Pro Bueno, A. \& Solbes, J. (2016). ¿Qué ciencias se enseñan y cómo se hace en las aulas de educación infantil? La visión de los maestros en formación inicial. Enseñanza de las ciencias, 34(3), 25-50. Doi: 10.5565/rev/ensciencias.1870

Ceballos, M. (2017). Aprovechamiento didáctico de los huertos escolares en centros de Sevilla. Enseñanza de las ciencias, (Extra-2017), 787-792.

Conde Campos, O., \& Pinzolas Torremocha, J. A. (2005). Los patios de recreo: espacios para la biodiversidad. Aula de Innovación Educativa, 140, 54-57.

Corraliza, J.A. \& Collado, S. (2011). La naturaleza cercana como moderadora del estrés infantil. Psichotema, 23, 221-226.

Cutter-Mackenzie, A., Edwards, S., Moore, D. \& Boyd, W. (2014). Young children's play and environmental education in early childhood education. Springer Science \& Business Media.

De Pro Chereguini, A., de Pro Bueno, A. \& Rodríguez Moreno, J. (2018). ¿Qué visión de la enseñanza de las ciencias se transmite a nuestros estudiantes en las Prácticas de Enseñanza en el Grado de Educación Infantil? En: C. Martínez Losada \& S. García Barros (eds.) 28 Encuentros de Didáctica de las Ciencias Experimentales, (pp. 601-606). A Coruña: Universidade da Coruña.

Escutia, M. (2009). El huerto escolar ecológico. Barcelona: Graó.

Eugenio Gozalbo, M. \& Aragón Núñez, L. (2016). Experiencias en torno al huerto ecológico como recurso didáctico y contexto de aprendizaje en la formación inicial de maestros/as de Infantil. Revista Eureka sobre Enseñanza y Divulgación de las Ciencias 13(3), 667-679.

Gadotti, M. (2002). Pedagogía de la Tierra. Buenos Aires: Siglo XXI.

García Gómez, J. \& Martínez Bernat, F.J. (2010). Cómo y qué enseñar de la biodiversidad en la alfabetización científica. Enseñanza de las ciencias, 28(2), 175-184.

Garrido Romero, J.M., Perales Palacios, F.J. \& Galdón Delgado, M. (2007). Ciencia para educadores. Madrid: Pearson Educación. 
Kesebir, S. \& Kesebir, P. (2017). A growing disconnection from nature is evident in cultural products. Perspectives on Psychological Science, 12(2), 258-269. Doi: 10.1177/1745691616662473

Loxley, P. (2018). Practical Ideas for Teaching Primary Science. Inspiring Learning and Enjoyment. Londres: Routledge.

Marandino, M. \& Díaz Rocha, P.E. (2011). La Biodiversidad en exposiciones inmersivas de museos de ciencias: implicaciones para educación en museos. Enseñanza de las Ciencias, 29(2), 221-236.

Mérida Serrano, R., Torres-Porras, J. \& Alcántara Manzanares, J. (eds.) (2017). Didáctica de las ciencias experimentales en educación infantil. Un enfoque práctico. Madrid: Editorial Síntesis.

Miguélez, M., Ansedes, B., Vidal, M. \& Membiela, P. (2017). Un proyecto centrado en los insectos palo. Aula de Infantil, 90, 35-38.

Perales Palacios, F.J. (2018). El área de Didáctica de las Ciencias Experimentales en España: entre la tribulación y la esperanza. Ápice. Revista de Educación Científica, 2(2), 1-14. Doi: 10.17979/arec.2018.2.2.3915

Prévot-Julliard, A. C., Julliard, R. \& Clayton, S. (2015). Historical evidence for nature disconnection in a 70-year time series of Disney animated films. Public Understanding of Science, 24(6), 672-680. Doi: $10.1177 / 0963662513519042$

Pyle, R. M. (2003). Nature matrix: Reconnecting people and nature. Oryx, 37, 206-214. Doi: $10.1017 /$ S0030605303000383

Romón Salinas, C. (2014). Guía del huerto escolar (Nueva edición). Madrid: Editorial Popular.

Ruiz Heredia, A. R. (2008). Educación, Medio Ambiente y Didáctica del Entorno Teoría y prácticas. Madrid: Editorial Popular.

Sanz Alonso, J., Sistiaga Poveda, M., Urkidi Elorrieta, P., Andoño Erdozain, I. \& Orbegozo Rezola, K. (2018). Educar en la naturaleza en infantil: modelo de evaluación para un bosque escuela. En: C. Martínez Losada \& S. García Barros (eds.) 28 Encuentros de Didáctica de las Ciencias Experimentales, (pp. 327-331). A Coruña: Universidade da Coruña.

Sauvé, L. (2017). Education as life. En: B. Jickling \& S. Sterling (eds.), Post-Sustainability and Environmental Education: Remaking Education for the Future (pp. 111-124). Cham (Suiza): Palgrave Macmillan (Springer).

Torres-Porras, J., Alcántara, J., Arrebola, J.C., Rubio, S.J. \& Mora, M. (2017). Trabajando el acercamiento a la naturaleza de los niños y niñas en el Grado de Educación Infantil. Crucial en la sociedad actual. Revista Eureka sobre Enseñanza y Divulgación de las Ciencias, 14(1), 258-270.

Varela-Losada, M., Vega-Marcote, P., Pérez-Rodríguez, U., \& Álvarez-Lires, M. (2016). Going to action? A literature review on educational proposals in formal Environmental Education. Environmental Education Research, 22(3), 390-421. doi: 10.1080/13504622.2015.1101751

Vega, S. (2006). Ciencia 0-3: laboratorios de ciencias en la escuela infantil. Barcelona: Graó.

Vega, S. (2012). Ciencia 3-6: laboratorios de ciencias en la escuela infantil. Barcelona: Graó. 
Vidal López, M. (2014). La enseñanza de las ciencias centrada en actividades prácticas de laboratorio. Un estudio de caso en la formación inicial de maestras y maestros de Educación Infantil. Tesis doctoral, Ourense: Universidad de Vigo.

Wells, N. M. \& Lekies, K. S. (2006). Nature and the life course: Pathways from childhood nature experiences to adult environmentalism. Children Youth and Environments, 16(1), 1-24.

\section{Agradecimientos}

Los autores desean agradecer al personal docente de los centros educativos asturianos la participación en este estudio, las facilidades en el contacto y aclaraciones, así como la contestación del cuestionario. 